Article

\title{
Investigation on Thrust and Moment Coefficients of a Centrifugal Turbomachine ${ }^{+}$
}

\author{
Bo Hu *, Dieter Brillert, Hans Josef Dohmen and Friedrich-Karl Benra \\ Department of Mechanical Engineering, University of Duisburg-Essen, 47057 Duisburg, Germany; \\ dieter.brillert@uni-due.de (D.B.); hans-josef.dohmen@uni-due.de (H.J.D.); friedrich.benra@uni-due.de (F.-K.B.) \\ * Correspondence: bo.hu.1987@stud.uni-due.de; Tel.: +49-203-379-1703 \\ + This paper is an extended version of our paper published in Proceedings of the 17th International Symposium \\ on Transport Phenomena and Dynamics of Rotating Machinery (ISROMAC 2017), Paper No. 77.
}

Received: 9 February 2018; Accepted: 4 April 2018; Published: 11 April 2018

\begin{abstract}
In radial pumps and turbines, the centrifugal through-flow in both the front and the back chambers is quite common. It strongly impacts the core swirl ratio, pressure distribution, axial thrust and frictional torque. In order to investigate these relationships experimentally, a test rig was designed at the University of Duisburg-Essen and described in this paper. Based on both the experimental and numerical results, correlations are determined to predict the impacts of the centrifugal through-flow on the core swirl ratio, the thrust coefficient and the moment coefficient. Two correlations respectively are determined to associate the core swirl ratio with the local through-flow coefficient for both Batchelor type flow and Stewartson type flow. The correlations describing the thrust coefficient and the moment coefficient in a rotor-stator cavity with centripetal through-flow (Hu et al., 2017) are modified for the case of centrifugal through-flow. The Daily and Nece diagram distinguishing between different flow regimes in rotor-stator cavities is extended with a through-flow coordinate into 3D. The achieved results provide a comprehensive data base which is intended to support the calculation of axial thrust and moment coefficients during the design process of radial pumps and turbines in a more accurate manner.
\end{abstract}

Keywords: rotor-stator cavity; centrifugal through-flow; core swirl ratio; pressure; thrust coefficient; moment coefficient

\section{Introduction}

Rotor-stator cavities are common elements in radial pumps and turbines. The schematic diagram of a rotor-stator cavity is shown in Figure 1. 


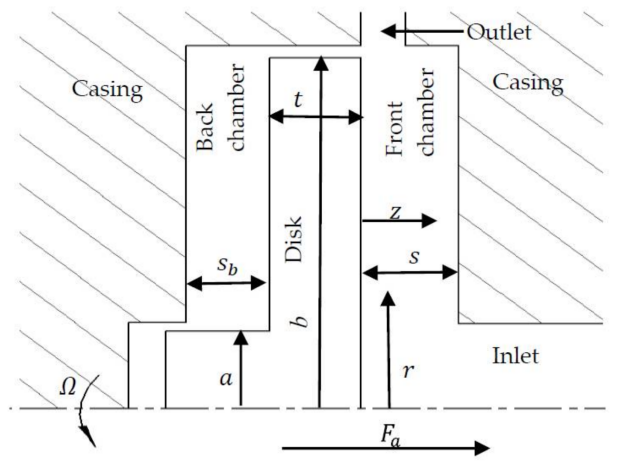

Figure 1. Geometry of a rotor-stator cavity. $a$ : hub radius; $b$ : outer radius of the disk; $F_{a}$ : axial thrust; $r$ : radial coordinate; $s$ : axial gap of the front chamber; $s_{b}$ : axial gap of the back chamber; $t$ : thickness of the disk; $z$ : axial coordinate; $\Omega$ : angular velocity of the disk.

Von Kármán [1], Cochran [2] and Daily and Nece [3] studied the flow around a rotating disk. Daily et al. [4], Bayley and Owen [5], Haynes and Owen [6] studied the flow structure theoretically and experimentally in an unshrouded rotor-stator cavity with centrifugal through-flow. By pressure measurements, Phadke and Owen [7] tested the impact of radial-clearance seals on the cavity flow in an air-cooled rotor-stator cavity with centrifugal through-flow. Launder et al. [8] investigated the instability patterns of the turbulent flow in a rotor-stator cavity. Will et al. [9-11] investigated the flow structure in the side chamber of a radial pump based on numerical simulation. Coren et al. [12], Long et al. [13] and Barabas et al. [14] conducted the experimental investigations on the cavity flow with large global circumferential Reynolds number Re. Daily et al. [3] gave an empirical correlation for the distribution of the core swirl ratio $K$ (the ratio of the tangential velocity of fluid to that of the disk at $\zeta=0.5$ ) along the radius of the disk in a rotor-stator cavity with centrifugal non-pre-swirl through-flow. From LDA (Laser Doppler Anemometer) measurements, Poncet et al. [15-17] and Debuchy et al. $[18,19]$ respectively correlated equations for $K$ according to the local flow rate coefficient $C_{q r}$. Lock et al. [20] investigated the core swirl ratio $K$ and the heat transfer rate in a rotor-stator cavity with pre-swirl nozzles which delivers the cooling air to the turbine blade in gas turbines.

The thrust coefficient $C_{F}$ and the moment coefficient $C_{M}$ are two major parameters to be considered in radial pumps and turbines and many accomplishments have been made on these areas. On the basis of the results of Goldstein [21], an implicit relation for $C_{M}$ under turbulent flow conditions was conducted by Schlichting and Gersten [22]. Kurokawa et al. [23-25] investigated $C_{F}$ and $C_{M}$ in an enclosed rotor-stator cavity with centrifugal or centripetal through-flow. From the axial thrust measurements, Hu et al. [26] determined a correlation for $C_{F}$ in a rotor-stator cavity with centripetal through-flow. Based on the simulation results of velocity profiles, a part of the 2D Daily and Nece diagram was extended into 3D with a third axis of through-flow coefficient $C_{D}{ }^{\prime}$. The torque measurements allowed the determination of the two correlations to describe the impact of $C_{D}{ }^{\prime}, \operatorname{Re}$ and the non-dimensional axial gap width $G$ on $C_{M}$ for regime III (merged disk boundary layer and wall boundary layer, namely Couette type flow) and regime IV (separated disk boundary layer and wall boundary layer, namely Batchelor type flow).

This present study is focused on the impact of centrifugal through-flow on $C_{F}$ and $C_{M}$, so that the influence of both the centripetal [26] and the centrifugal through-flow can be compared. Then the axial thrust $F_{a}$ and the frictional torque $M$ can be better predicted. The definitions of the significant non-dimensional parameters applied in this study are given in Equation (1a)-(1k).

$$
\begin{aligned}
R e & =\frac{\Omega \cdot b^{2}}{v} \\
R e_{\varphi} & =\frac{\Omega \cdot r^{2}}{v}
\end{aligned}
$$




$$
\begin{gathered}
G=\frac{s}{b} \\
C_{D}{ }^{\prime}=\frac{\dot{m}}{\mu \cdot b} \\
C_{F}=\int_{a}^{b} \frac{2 \cdot \pi \cdot\left(p_{b}-p\right) \cdot r \mathrm{~d} r}{\rho \cdot \omega^{2} \cdot b^{4}} \\
C_{q r}=\frac{Q \cdot R e_{\varphi}^{0.2}}{2 \cdot \pi \cdot \Omega \cdot r^{3}} \\
\zeta=\frac{z}{s} \\
x=\frac{r}{b} \\
C_{M}=\frac{2 \cdot|M|}{\rho \cdot \Omega^{2} \cdot b^{5}} \\
p^{*}=\frac{p}{\rho \cdot \Omega^{2} \cdot b^{2}} \\
C_{p}=p^{*}(x=1)-p^{*}(x)
\end{gathered}
$$

\section{Theoretical Analysis}

In a rotor-stator cavity with centrifugal through-flow, rotation dominant flow (Batchelor or Couette type flow) and through-flow dominant flow (Stewartson type flow) are quite common. Qualitative profiles of both non-dimensional velocities $V_{\varphi}$ and $V_{r}$ along $\zeta$ are depicted in Figure 2. Based on the LDA measurements by Poncet et al. [15-17], the transition zone between the rotation dominant flow and the through-flow dominant flow is found in the parameter range $0.02 \leq C_{q r} \leq 0.03$.

Rotation dominant flow: $C_{q r}<0.02$

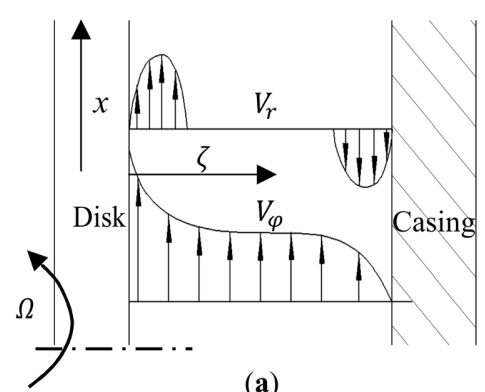

(a)

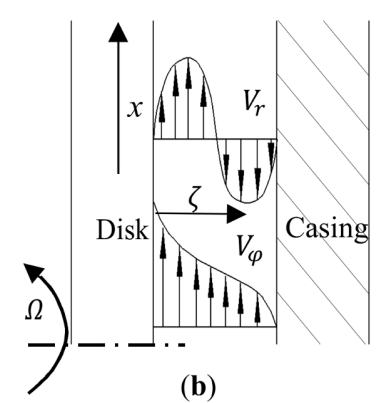

(b)
Through-flow dominant flow: $C_{q r}>0.03$

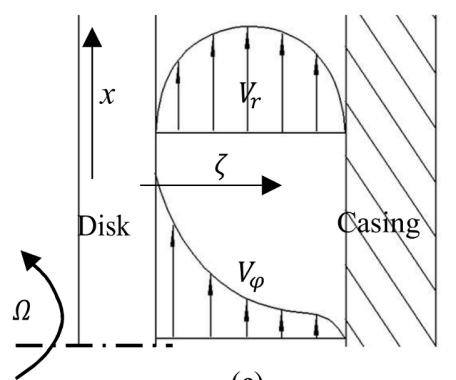

(c)

Figure 2. Velocity profiles for both the rotation dominant flow and the through-flow dominant flow: (a) Batchelor type flow; (b) Couette type flow and (c) Stewartson type flow. $C_{q r}$ : local flow rate coefficient; $V_{r}$ : non-dimensional radial velocity; $V_{\varphi}$ : non-dimensional tangential velocity; $x$ : non-dimensional radial coordinate; $\zeta$ : non-dimensional axial coordinate; $\Omega$ : angular velocity of the disk.

To predict the axial thrust, the pressure distribution along the radius of the disk should be estimated. With increasing $C_{q r}$, the flow type may change from Batchelor or Couette type flow to Stewartson type flow. The predictions of $K$ for the rotation dominant flow can be conducted with Equation (2a) (by Poncet et al. [15,17]) or Equation (2b) (by Debuchy et al. [18,19]). In addition, a correlation of $K$ for the through-flow dominant flow is derived by Poncet et al. $[16,17]$ and written in Equation (2c). Results of the three equations are depicted in Figure 3a. Figure 3b depicts the narrow transition zone from Batchelor or Couette type flow to Stewartson type flow with the increase of $C_{q r}$. 
Equation (2b) is chosen in this paper to predict the values of $K$ for Batchelor or Couette type flow in a wider range of $C_{q r}$.

$$
\begin{gathered}
K=2 \cdot\left(-5.9 \cdot C_{q r}+0.63\right)^{\frac{5}{7}}-1 ;-0.2 \leq C_{q r}<0.02 \\
K=\left[\frac{-8.85 \cdot C_{q r}+0.5}{e^{\left(-1.45 \cdot C_{q r}\right)}}\right]^{\frac{5}{4}} ;-0.5 \leq C_{q r}<0.02 \\
K=0.032+0.32 \times e^{\frac{-C_{q r}}{0.028}} ; C_{q r}>0.03
\end{gathered}
$$

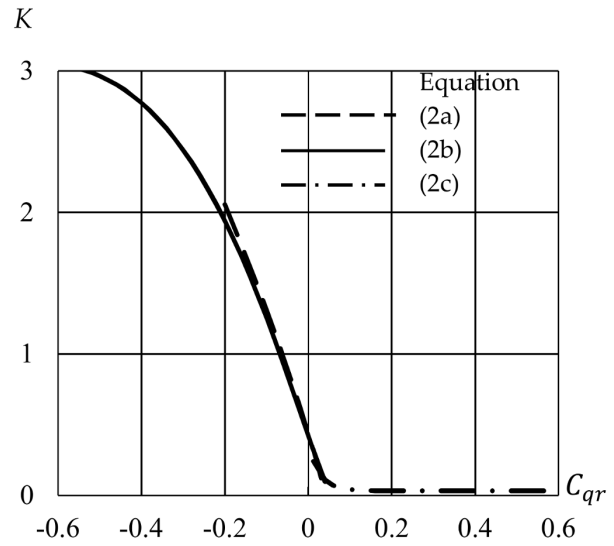

(a)

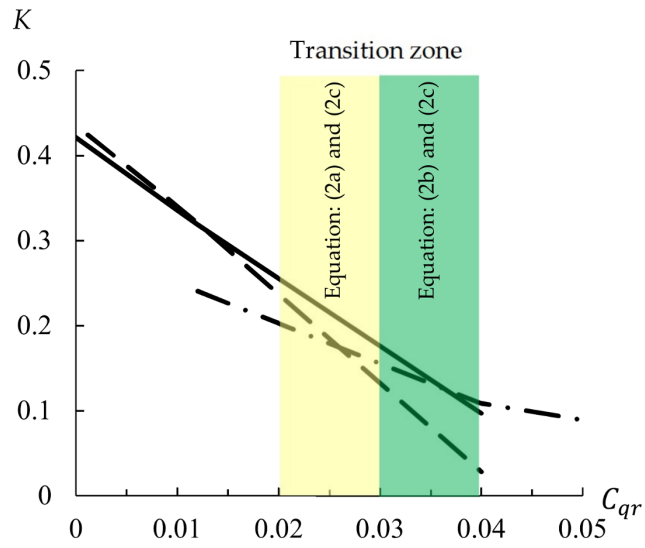

(b)

Figure 3. $K\left(C_{q r}\right)$ curves: (a) Equation (2a)-(2c) and (b) near the transition zone of the rotation dominant flow and the through-flow dominant flow. $K$ : core swirl ratio at $\zeta=0.5 ; C_{q r}$ : local flow rate coefficient.

Numerous researches, such as those by Kurokawa et al. [23-25] and Poncet et al. [15-17], show that the pressure distribution along the radius of the disk can be estimated with the core swirl ratio $K$, as a function of $C_{q r}$, by Equation (3a) both with and without through-flow neglecting the turbulent wall shear stress. In Will et al. [9-11], Equation (3b) is determined to evaluate the pressure distribution along the radius of the disk in a rotor-stator cavity with through-flow for incompressible, steady flow. Along the radius of the disk, the cross-sectional area of the cavity is like a diffusor. Consequently, the pressure must also change since the velocity changes in the radial direction according to the continuity equation.

$$
\begin{gathered}
\frac{\partial p}{\partial r}=\rho \cdot K\left(C_{q r}\right)^{2} \cdot \Omega^{2} \cdot r \\
\frac{\partial p}{\partial r}=\rho \cdot K\left(C_{q r}\right)^{2} \cdot \Omega^{2} \cdot r+\frac{\rho \cdot Q^{2}}{4 \cdot \pi^{2} \cdot s^{2} \cdot r^{3}}
\end{gathered}
$$

Based on Equation (3b), the pressure along the radius of the disk can be calculated with Equation (4) based on the values of $K$, which is a variable along the radius of the disk. A simplification is made as follows: $K$ is a fixed value every $1 \mathrm{~mm}$ in radial direction. Then, the approximate pressure distribution along the disk can be calculated with Equation (4). The parameter $p_{b}$ represents the pressure at the non-dimensional radius $x=1$. Due to the construction of the test rig, there is no pressure tube at $x=1$. The closest pressure tube in the front cavity is at $x=0.955$. Hence, the value of $p_{b}$ is calculated combining the measured pressure at $x=0.955$ with Equation (4).

$$
p(r)=p_{b}+\int_{b}^{r} \rho \cdot K\left(C_{q r}\right)^{2} \cdot \Omega^{2} \cdot r d r+\frac{\rho \cdot Q^{2}}{8 \cdot \pi^{2} \cdot s^{2}}\left(\frac{1}{b^{2}}-\frac{1}{r^{2}}\right)
$$


where

$$
\begin{gathered}
\int_{b}^{r} \rho \cdot K\left(C_{q r}\right)^{2} \cdot \Omega^{2} \cdot r d r \approx \frac{\rho}{2} \cdot \Omega^{2} \cdot \sum_{0}^{c-1} K_{r_{i+1}}^{2} \cdot\left(r_{i}^{2}-r_{i+1}^{2}\right) ; \\
r_{i}-r_{i+1}=-0.001(\mathrm{~m}) ; \\
c=\frac{b-r}{0.001(\mathrm{~m})} .
\end{gathered}
$$

The difference of the forces on both sides of the disk is the main source for the axial thrust $F_{a}$, calculated with Equation (5a). In Equation (5b), $F_{a f}$ and $C_{F f}$ respectively represent the force and the thrust coefficient at the front surface of the disk shown in Figure 1, while $F_{a b}$ and $C_{F b}$ in Equation (5c) are those at the back surface of the disk (in the back chamber). The parameter $a$ represents the radius of the hub (see Figure 1). The back chamber $(G=0.072)$, shown in Figure 1, is viewed as an enclosed cavity. The values of $C_{F b}$ are obtained when $C_{D}{ }^{\prime}=0$ and the axial gaps of the both cavities are of the same size for different Reynolds numbers $R e$ (these conditions yield to the conclusion: $C_{F f}=C_{F b}$ ). After obtaining those values, the values of $C_{F f}$ with different values of $C_{D}{ }^{\prime}$ can be calculated with Equation (6).

$$
\begin{gathered}
F_{a}=F_{a b}-F_{a f} \\
F_{a f}=\pi \cdot p_{b} \cdot b^{2}-C_{F f} \cdot \rho \cdot \Omega^{2} \cdot b^{4} \\
F_{a b}=\pi \cdot p_{b} \cdot\left(b^{2}-a^{2}\right)-C_{F b} \cdot \rho \cdot \Omega^{2} \cdot\left(b^{4}-a^{4}\right) \\
C_{F f}=\frac{F_{a}+C_{F b} \cdot \rho \cdot \Omega^{2}\left(b^{4}-a^{4}\right)+\pi p_{b} a^{2}}{\rho \cdot \Omega^{2} \cdot b^{4}}
\end{gathered}
$$

\section{Numerical Simulation Set-Up}

To predict the flow in the cavity, numerical simulations are carried out using the academic ANSYS CFX 14.0 (ANSYS Inc., Canonsburg, PA, USA). Considering the axial symmetry of the cavity, a 15 degrees segment of the whole domain is modeled and a rotational periodic boundary condition is applied. Structured meshes are generated with ICEM 14.0. The domain for numerical simulation when $G=0.072$ is depicted with yellow color in Figure 4 . The simulation type is set as steady state. The deviations between the pressure measurements and the simulation results of pressure from the SST $k-\omega$ turbulence model in combination with the scalable wall are less than $1 \%$ in a rotor-stator cavity operated with air (Barabas et al. [14]). Hence, in this study, the same turbulence model and wall functions are used. The discretization scheme is set as second order upwind. A no-slip wall condition is defined for all walls. The boundary condition at the inlet is static pressure and at the outlet is mass flow. The values of pressure at the inlet are set according to the measured pressure at the pump outlet. The convergence criteria are set as $10^{-5}$ in maximum type. The maximum value of $y^{+}$for all the simulation domains in the rotor-stator cavities is 4.3. Mesh sensitivity analyses are accomplished for all the simulation models. 


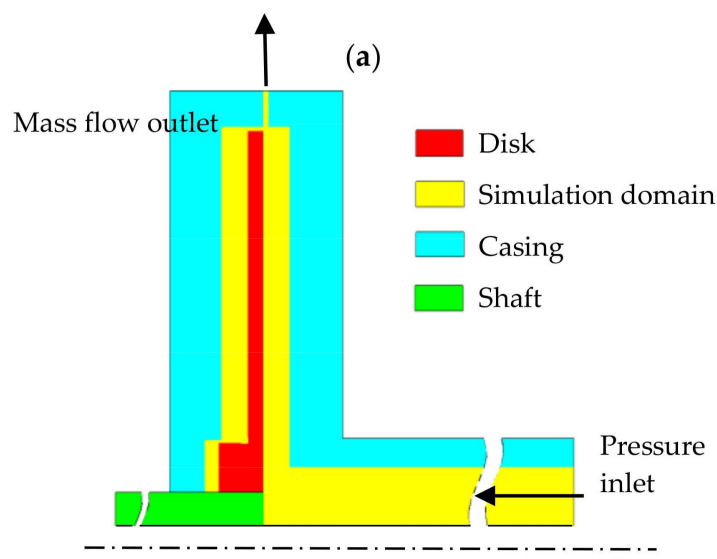

(b)

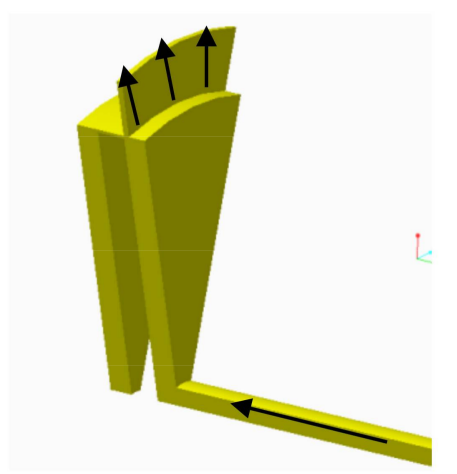

Figure 4. Domain for numerical simulation at $G=0.072$ : (a) Cross section of the cavity model and (b) Simulation domain.

In Figure 5, the results from numerical simulations for $C_{p}$ along the radius are compared with those by Poncet et al. [16] at $R e=4.15 \times 10^{6}$ and $G=0.036$. The reference pressures are taken at $x=0.92$ instead of $x=1$. The differences between the $C_{p}$ from numerical simulations and pressure measurements are less than $6 \%$. Hence, the numerical simulation set-up should be reasonable.

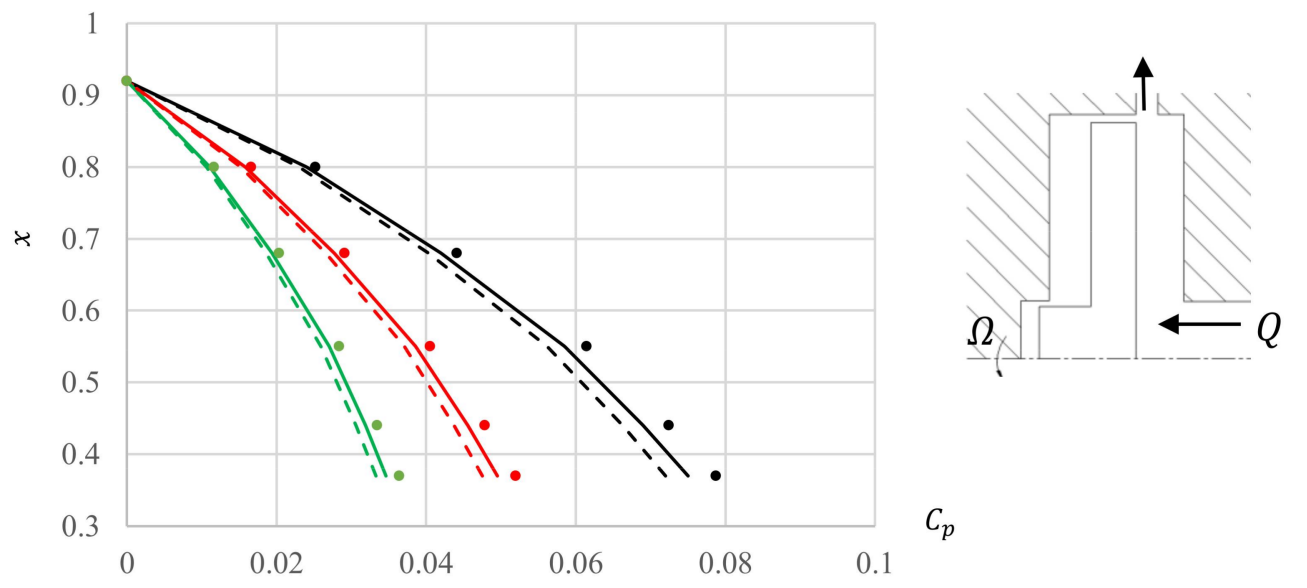

Figure 5. Comparison of radial pressure distribution for $R e=4.15 \times 10^{6}$ and $G=0.036$. $C_{p}$ : pressure coefficient; $G$ : non-dimensional axial gap; $Q$ : volumetric through-flow rate; $x$ : non-dimensional radial coordinate; $\Omega$ : angular velocity of the disk. Colors: Black: $C_{D}{ }^{\prime}=0$, Red: $C_{D}{ }^{\prime}=2579$, Green: $C_{D}{ }^{\prime}=5159$; Solid lines: RSM from Poncet et al. [16]; Dashed lines: Simulation results (SST $\left.k-\omega\right)$; Points: Measurements by Poncet et al. [16].

\section{Test Rig Design and Experimental Set-Up}

The cross section of the test rig is illustrated in Figure 6a. The view along the " $A$ " direction is sketched in Figure 6b. The shaft sealing at the back cavity is depicted in Figure 6c. The test rig is supplied with water by a pump system shown in Figure $6 \mathrm{~d}$ and the shaft is driven by an electrical motor. A frequency converter is used to adjust the speed of rotation $n$ (0 to $2500 \mathrm{rpm}$ ) with the absolute error of $7.5 \mathrm{rpm}$. In this study, only the axial gap of the front chamber is varied by installing six sleeves at different lengths. At the outlet, there are 24 passages in the guide vane shown in Figure $6 \mathrm{~b}$, instead of it being entirely open at the periphery. The radial directed guide vane has an area of $5.4 \times 10^{-5} \mathrm{~m}^{2}$ at the entrance of each passage (see Figure $6 \mathrm{~b}$ ). The transducers in the test rig include 12 pressure tubes in the front chamber, a torque meter and three tension compression transducers. A thrust plate is 
fixed by a ball bearing and a nut from each side to convey the axial thrust to the tension compression transducers. A linear bearing is utilized to minimize the frictional resistance during the axial thrust measurements. The axial thrust transducers are recalibrated after changing the axial gap width of the front chamber. The measured surface roughness $R_{z}$ of the disk is $1 \mu \mathrm{m}$. The values of $R_{z}$ on all other wet surfaces are below $1.6 \mu \mathrm{m}$. The rest of the parameters of the experiments in this study are given in Table 1.

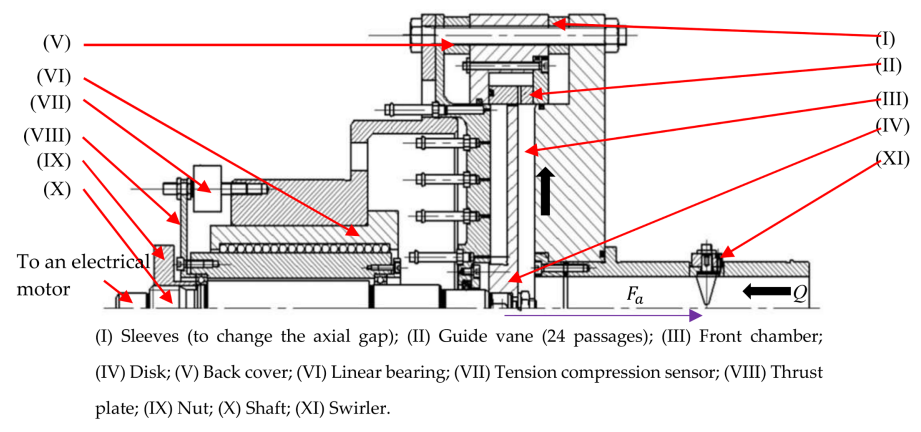

(a)

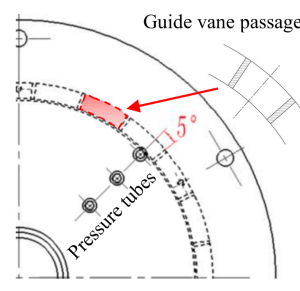

(b)

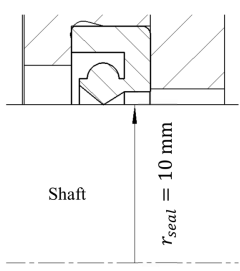

(c)

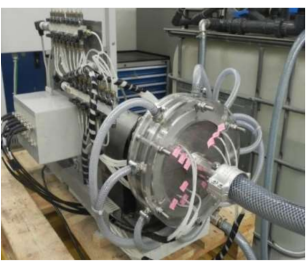

(d)

Figure 6. Test rig design: (a) Cross section of the test rig; (b) View along " $A$ " direction; (c) Shaft sealing and (d) Test rig. $F_{a}$ : axial thrust; $r_{\text {seal }}$ : radius of shaft seal.

Table 1. Parameters of the experiments.

\begin{tabular}{ccccccc}
\hline $\boldsymbol{b}(\mathbf{m m})$ & $\boldsymbol{n}(\mathrm{rpm})$ & $Q\left(\mathrm{~m}^{3} / \mathrm{s}\right)$ & $\boldsymbol{s}(\mathbf{m m})$ & $\boldsymbol{s}_{\boldsymbol{b}}(\mathbf{m m})$ & $\boldsymbol{a}(\mathbf{m m})$ & $\boldsymbol{t}(\mathbf{m m})$ \\
\hline 110 & 0 to 2500 & 0 to $5.56 \times 10^{-4}$ & 2 to 8 & 8 & 23 & 10 \\
\hline
\end{tabular}

$a$ : hub radius; $b$ : outer radius of the disk; $n$ : speed of rotation; $Q$ : volumetric through-flow rate; $s$ : axial gap of the front chamber; $s_{b}$ : axial gap of the back chamber; $t$ : thickness of the disk.

The measurements of the axial thrust coefficient include two steps. The cavities for each step are depicted in Figure 7. The first step, depicted in Figure 7a, is to measure the axial force imposed by the drive end of the motor when the shaft without the disk is rotating at different speed of rotation in the air. For the second step, all the results are modified by subtracting the above values obtained at the first step corresponding to the same speed of rotation. The shaft end (with the area of $\Omega \cdot r_{e}{ }^{2}$ ) in Figure $7 \mathrm{~b}$ is considered as a part of front surface and the geometry of the nut is ignored. Then, the values of thrust coefficient on a single surface can be calculated with Equation (6). 


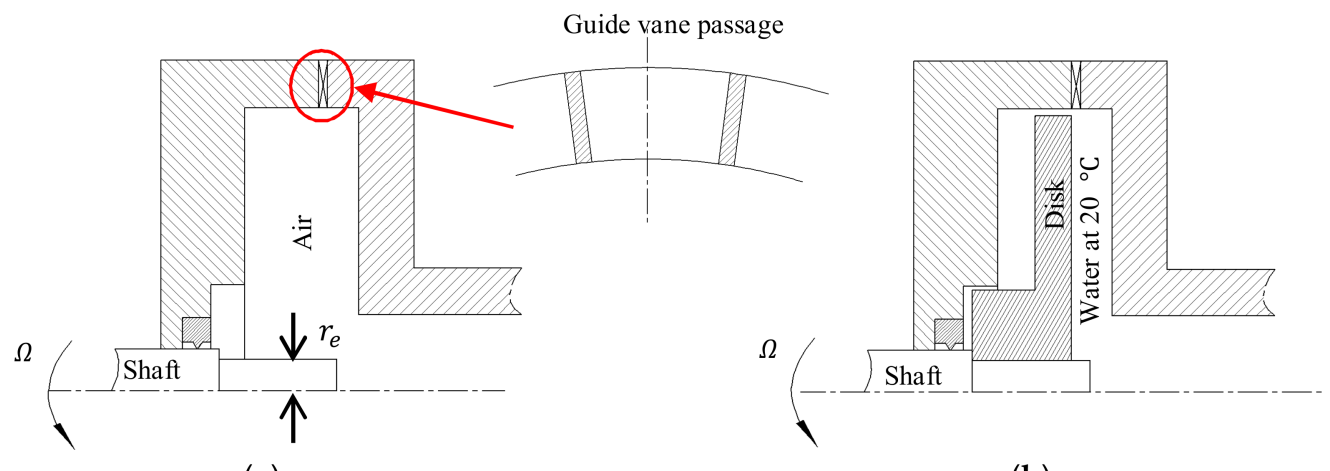

(a)

(b)

Figure 7. Cavities to measure the axial thrust: (a) Step 1 and (b) Step 2. $r_{e}$ : radius of the shaft end; $\Omega$ : angular velocity of the disk.

To compute the torque precisely, the frictional torque of the shaft needs to be subtracted. Therefore, measurements without the disk and at different rotating speed are conducted. The values of $C_{M}$ at the back surface are obtained when $C_{D}{ }^{\prime}=0$ and the axial gaps of the both cavities are of the same size for different Reynolds numbers $R e$.

The relative error, noted as $\mathrm{e}_{T}$, of the pressure transducers is $1 \%$ (full scale, FS). The value of $\mathrm{e}_{T}$ for the torque transducer is $0.1 \%$ (FS) and for the axial thrust transducers is $0.5 \%$ (FS). All the experimental results are an ensemble average of 1000 samples. The measuring range $\left(M_{r}\right)$ of the torque meter is 0 to $10 \mathrm{Nm}$. The measuring range of the pressure transducers is 0 to 2.5 bar (absolute pressure). The measuring range of the thrust transducers is -100 to $100 \mathrm{~N}$. The uncertainties of the measured results, noted as $\Delta N$, are summarized in Table 2 (Hu et al. [26]).

Table 2. Uncertainty analysis for the measurements.

\begin{tabular}{cccccc}
\hline & $\boldsymbol{p}$ (Bar) & $\boldsymbol{F}_{\boldsymbol{a}}(\mathbf{N})$ & $\boldsymbol{M} \mathbf{( N m )}$ & $\boldsymbol{R} \boldsymbol{e}$ & $\boldsymbol{C}_{\boldsymbol{D}}{ }^{\prime}$ \\
\hline$\Delta N$ & $4.04 \times 10^{-4}$ & $2.43 \times 10^{-2}$ & $3.00 \times 10^{-4}$ & $9.01 \times 10^{-4}$ & 4.1 \\
\hline
\end{tabular}

$\Delta N$ : uncertainty of the measured results; $p$ : pressure; $M$ : frictional torque; Re: global circumferential Reynolds number; $C_{D}{ }^{\prime}$ : through-flow coefficient.

\section{Results and Discussion}

\subsection{Core Swirl Ratio}

To evaluate the pressure distribution, the values of $K$ need to be estimated. Although some correlations are determined to predict the values of $K$ with centrifugal through-flow, such as Equations (2a)-(2c), it still exists an uncertainty on the impact of $G$ on $K$. In addition, the geometry of the cavity, especially at the outlet, does have large influence on $K$. Based on Equation (4), the pressure difference between the two adjacent pressure tubes number $e$ and number $e+1$ can be calculated with Equation (8). The parameter $\bar{K}$ represents the average value of $K$ between two adjacent pressure tubes. Twelve pressure tubes exist in the front chamber from $r=0.05 \mathrm{~m}(x=0.455)$ to $r=0.105 \mathrm{~m}$ $(x=0.954)$. Since the radial distances between the adjacent pressure tubes are small, the application of the average values of $K$ results in a small error only. The values of $C_{q r}$ for the experimental results are calculated with $r=\frac{r_{e}+r_{e+1}}{2}$. Therefore, the values of $K(K \approx \bar{K})$ can be verified combining the pressure measurements with Equation (9).

$$
p\left(r_{e}\right)=p\left(r_{e+1}\right)+\frac{1}{2} \cdot \rho \cdot \Omega^{2} \cdot \bar{K}^{2} \cdot\left(r_{e}^{2}-r_{\mathrm{e}+1}^{2}\right)+\frac{\rho \cdot Q^{2}}{8 \cdot \pi^{2} \cdot s^{2}}\left(\frac{1}{r_{e+1}{ }^{2}}-\frac{1}{r_{e}^{2}}\right)
$$




$$
\bar{K}=\sqrt{\frac{p\left(r_{e}\right)-p\left(r_{e+1}\right)-\frac{\rho \cdot Q^{2}}{8 \cdot \pi^{2} \cdot s^{2}}\left(\frac{1}{r_{e+1}{ }^{2}}-\frac{1}{r_{e}{ }^{2}}\right)}{\frac{1}{2} \cdot \rho \cdot \Omega^{2} \cdot\left(r_{e}{ }^{2}-r_{e+1}{ }^{2}\right)}}
$$

On the basis of the results from both numerical simulations and pressure measurements, Equation (10) is determined to describe the impacts of $G$ and $C_{q r}$ on $K$. For the rotation dominant flow, the values of $K$ follow the analytical law given in Equation (10a). For the through-flow dominant flow, the values of $K$ decrease exponentially with increasing $C_{q r}$ following Equation (10b). In Figure 8, the experimental results based on pressure measurements by Equation (9) are compared with those from numerical simulations and those from Equation (10). Relatively large errors occur only when $C_{q r}>0.01$, which can be attributed to the application of the average values of $K$ in and around the transition zone between the rotation dominant flow and the through-flow dominant flow. For the future work, more pressure taps are intended to be used at the low radius to reduce the error.

$$
\begin{aligned}
& \text { Rotation dominant flow }\left(-0.5 \leq C_{q r} \leq 0.032\right): K=\left(e^{-1.236 \cdot G}\right) \cdot\left[\frac{-8.85 \cdot C_{q r}+0.5}{\left.e^{\left(-1.45 C_{q r}\right.}\right)}\right]^{\frac{5}{4}} \\
& \text { Through - flow dominant flow }\left(C_{q r}>0.032\right): K=\left(e^{-1.236 \cdot G}\right) \cdot\left[0.032+0.32 \times e^{\left(\frac{-C_{q r}}{0.028}\right)}\right]
\end{aligned}
$$

The influence of $G$ on $K$ is weak according to the results in Figure 9. Poncet et al. [15-17] and Debuchy et al. $[18,19]$ neglected the impact of $G$ on $K$ with the results from LDA measurements. In most of the radial pumps and turbines, $G$ is a variable along the radius. A simplified correlation is required with good accuracy over the parameter range of $G$ in this study. By pressure measurements, Equation (11) is correlated to predict the values of $K$ when $G$ ranges from 0.018 to 0.072 . The results from Equation (11) are in good accordance with both the simulation results and the experimental results compared in Figure 9. In this paper, Equation (11) is applied in the calculation process of pressure instead of Equation (10).

$$
\begin{gathered}
\text { Rotation dominant flow }\left(-0.5 \leq C_{q r} \leq 0.032\right): K=0.97 \cdot\left[\frac{-10 \cdot C_{q r}+0.5}{e^{\left(-1.45 C_{q r}\right)}}\right]^{\frac{5}{4}} \\
\text { Through - flow dominant flow }\left(C_{q r}>0.032\right): K=0.85 \cdot\left[0.032+0.32 \times e^{\left(\frac{-C_{q r}}{0.028}\right)}\right]
\end{gathered}
$$

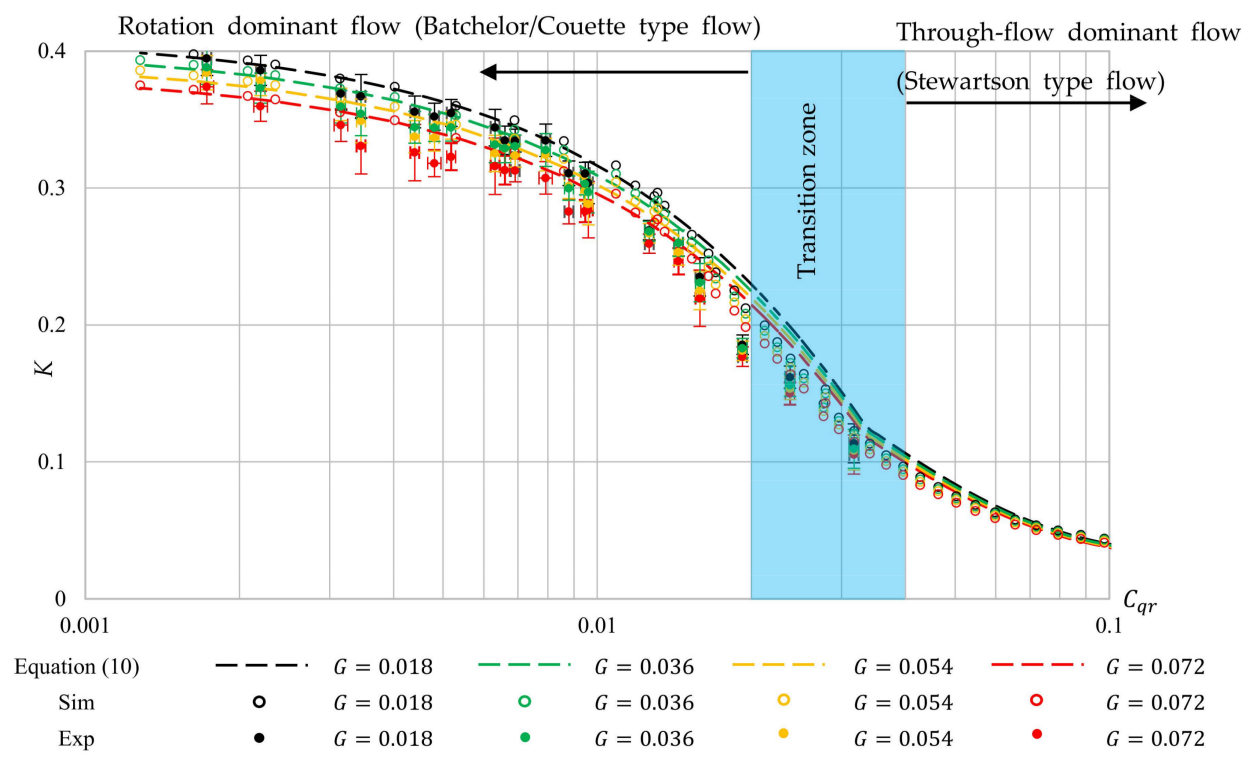

Figure 8. $K\left(C_{q r}\right)$ curves. $C_{q r}$ : local flow rate coefficient; $G$ : non-dimensional axial gap; $K$ : core swirl ratio. 


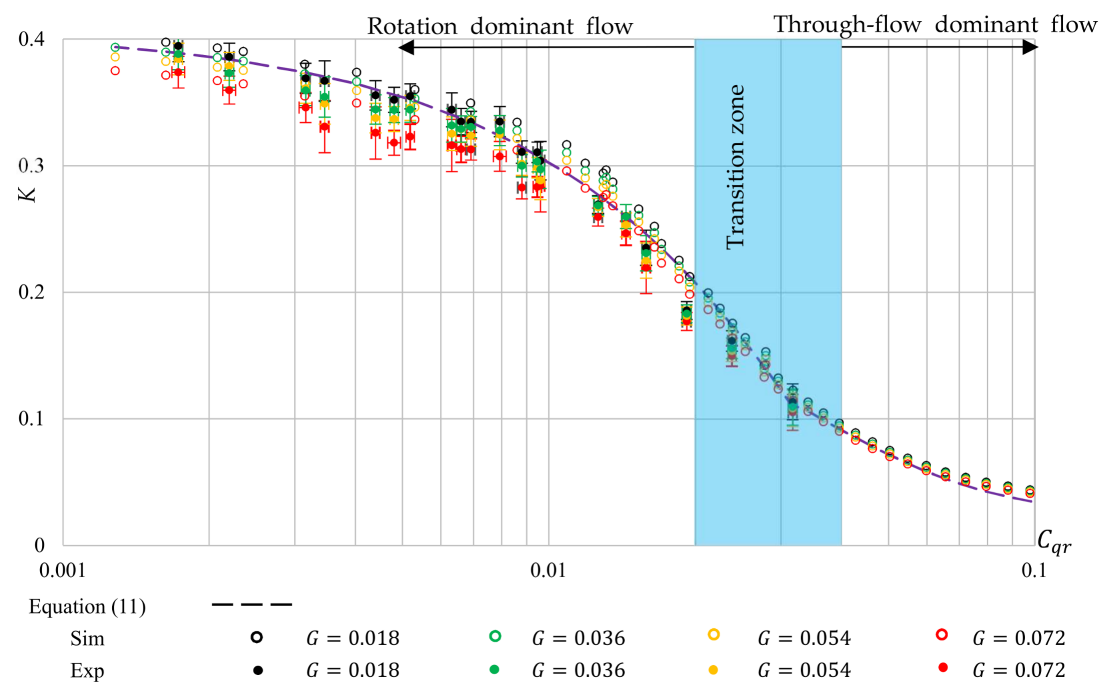

Figure 9. $K\left(C_{q r}\right)$ curve ignoring the impact of $G$. $C_{q r}$ : local flow rate coefficient; $G$ : non-dimensional axial gap; K: core swirl ratio.

Some values of $K$, however, do not fit to the results from Equation (11), especially at $x=0.955$ for wider gaps. A selection of the results is shown in Figure 10a. Near the outlet, an area change from the front cavity to the passage in the guide vane for $G=0.036,0.054$ and 0.072 occurs. The measured pressure at $x=0.955$ is strongly influenced by the geometry at the outlet of the test rig (see Figure 10b). Based on the simulation results, small vortices near the outer radius of the disk exist and therefore the measured values at $x=0.955$ are used only partially during the calculation of $K$.

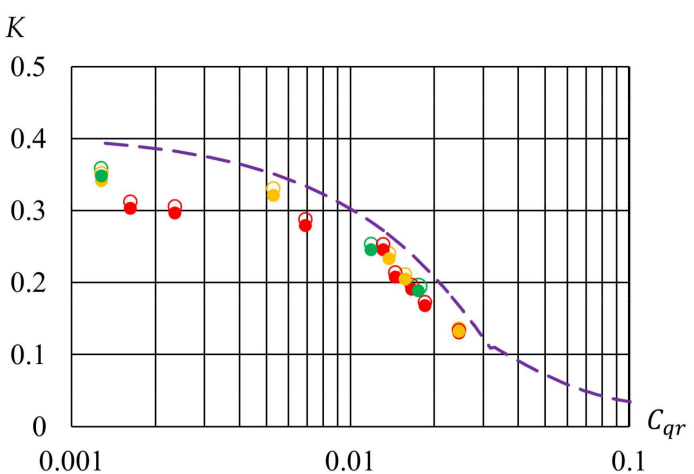

(a)

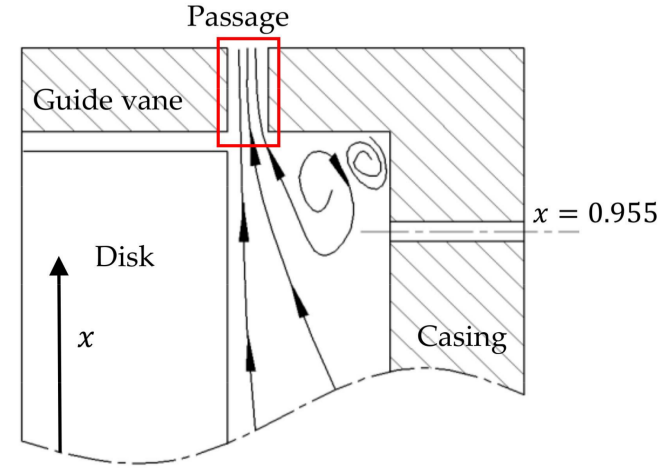

(b)

Figure 10. Large differences of $K$ attributed to the geometry near the outlet: (a) Part of the results do not fit Equation (11) and (b) Surface streamlines near the outlet (from numerical simulation). $C_{q r}$ : local flow rate coefficient; $K$ : core swirl ratio; $x$ : non-dimensional radial coordinate.

\subsection{Pressure Coefficient}

A reference pressure is taken at the non-dimensional radial coordinate $x=1$. The pressure values at $x=1$ are from Equation (4) based on the values of $K$ from Equation (11). The values of pressure coefficient $C_{p}$ are positive due to the pressure drop towards the shaft. In Figure 11, the values of $C_{p}$ are plotted versus $x$ varying $C_{D}{ }^{\prime}$ and $G$. The experimental results show that $C_{p}$ decreases with increasing $C_{D}{ }^{\prime}$ in general for shown $R e$ and $G$. The experimental results are in good agreement with those from the equations (difference less than $7 \%$ ). When $R e=2.79 \times 10^{6}$, the uncertainty of the $C_{p}$ is 
$1.3 \times 10^{-4}$, which is rather small compared to the measured results. Hence, the error bars are neglected in Figure 11d-f.

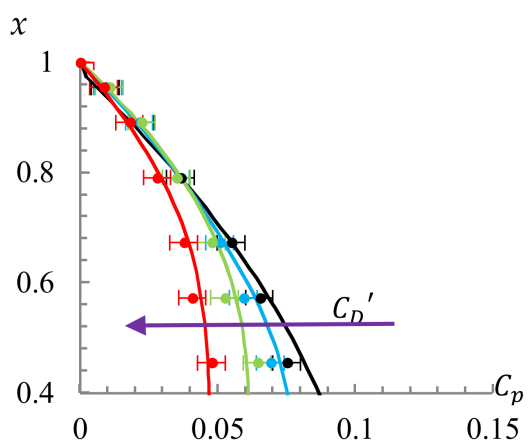

(a)

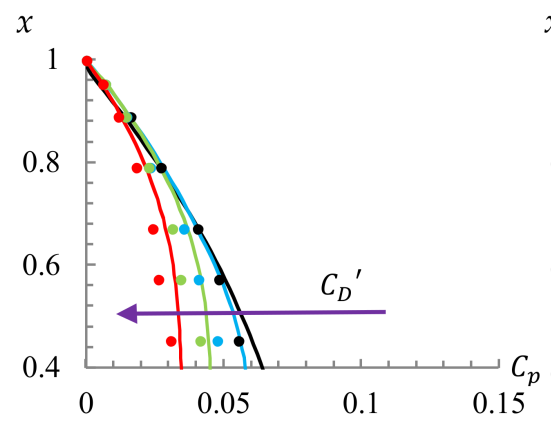

(d)

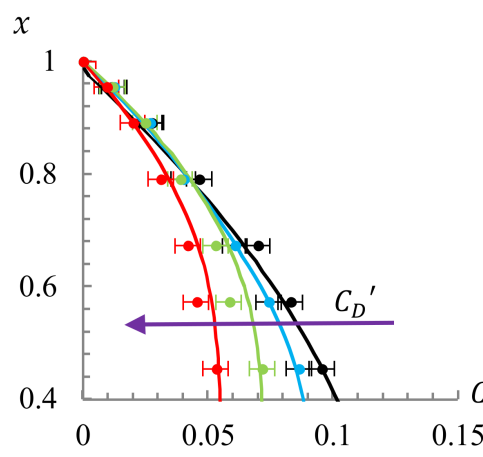

(b)

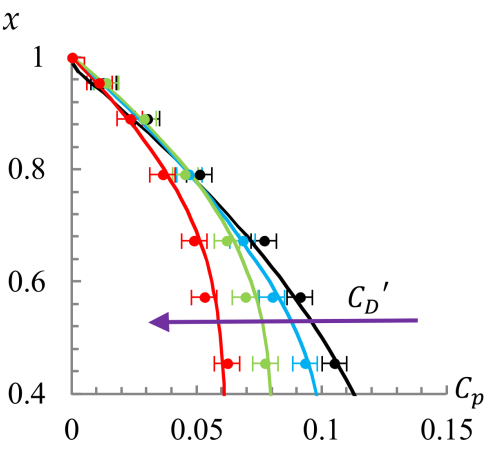

(c)

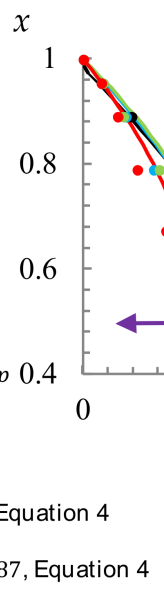

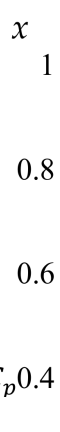

$x$

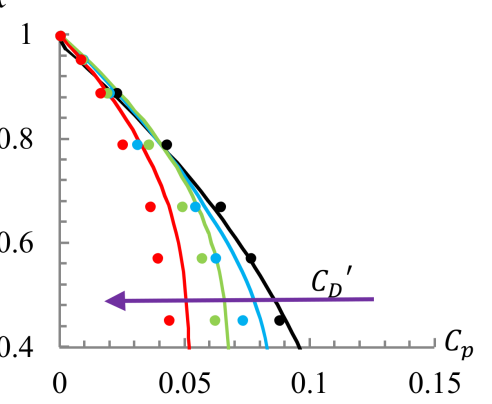

(e) (f) $\begin{aligned} \text { - } C_{D}{ }^{\prime} & =1262, \text { Exp } \\ \text { - } & C_{D}{ }^{\prime}=5050, \text { Exp }\end{aligned}$
$C_{D}{ }^{\prime}=1262$, Equation 4

$C_{D}{ }^{\prime}=5050$, Equation 4

Figure 11. Distribution of $C_{p}$ along the non-dimensional radius of the disk: (a) $R e=0.76 \times 10^{6}$, $G=0.072$; (b) $R e=0.76 \times 10^{6}, G=0.036$; (c) $\operatorname{Re}=0.76 \times 10^{6}, G=0.018$; (d) $R e=2.79 \times 10^{6}$, $G=0.072 ;(\mathbf{e}) \operatorname{Re}=2.79 \times 10^{6}, G=0.036 ;(\mathbf{f}) \operatorname{Re}=2.79 \times 10^{6}, G=0.018 . C_{D}{ }^{\prime}$ : through-flow coefficient; $C_{p}$ : pressure coefficient; $G$ : non-dimensional axial gap; $x$ : non-dimensional radial coordinate.

\subsection{Axial Thrust}

From the axial thrust measurements, an empirical correlation for the thrust coefficient in a rotor-stator cavity with centripetal through-flow is determined by Hu et al. [26]. Compared with the experimental results in this paper, the correlation is modified for centrifugal through-flow, written as:

$$
C_{F}=[0.0066 \cdot \ln (R e)-0.113] \cdot e^{\left(-0.00016 \cdot C_{D}{ }^{\prime}\right)} \cdot[0.122 \cdot \ln (G)-0.67]
$$

where

$$
\begin{gathered}
0.018<G<0.072 \\
C_{D}{ }^{\prime} \leq 5050 \\
R e \leq 3.17 \times 10^{6} .
\end{gathered}
$$

The comparison of the results of $C_{F}$ for different $G$ and $C_{D}{ }^{\prime}$ are shown in Figure 12. The parameter $B p$, which is the calculated thrust coefficient obtained from the pressure calculation along the radius of the disk, is calculated by combining the measured pressure with Equation (4) according to the values of $K$ (calculated with $C_{q r}$ every $1 \mathrm{~mm}$ along the radius for the rotation dominant flow or the through-flow dominant flow) from Equation (11). In the transition zone, the correlation of $K$ for rotation dominant flow is used. The experimental results of $C_{F}$ are in good agreement with those from the pressure 
calculation and Equation (12). The differences between the results of $C_{F}$ from Equation (12) and by axial thrust measurements are less than $5 \%$ in most of the measured points. Relatively large errors (smaller than $12.9 \%$ ) only occur at $R e=0.38 \times 10^{6}$. The values of $C_{F}$, which are smaller for wider axial gaps, decrease with increasing $\left|C_{D}{ }^{\prime}\right|$. As commonly understood, however, the values of $C_{F}$ increase with increasing $\left|C_{D}{ }^{\prime}\right|$ in a rotor-stator cavity with centripetal through-flow ( $C_{D}^{\prime}$ is negative) [26].

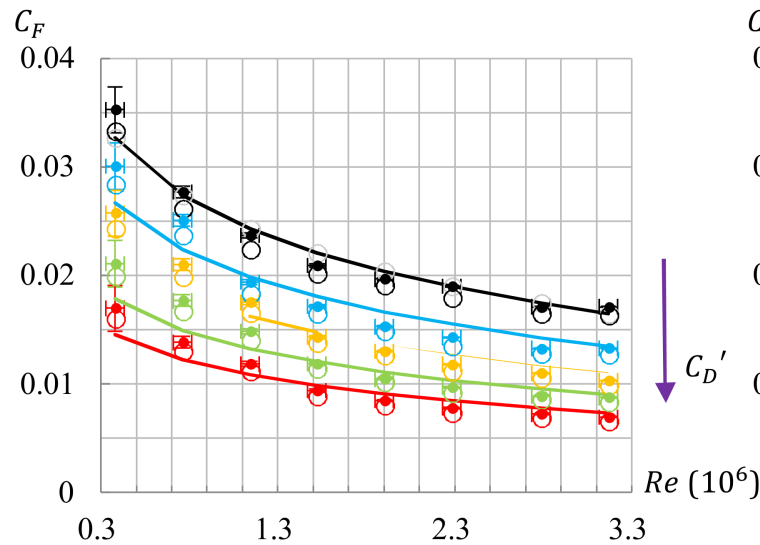

(a)

$C_{F}$

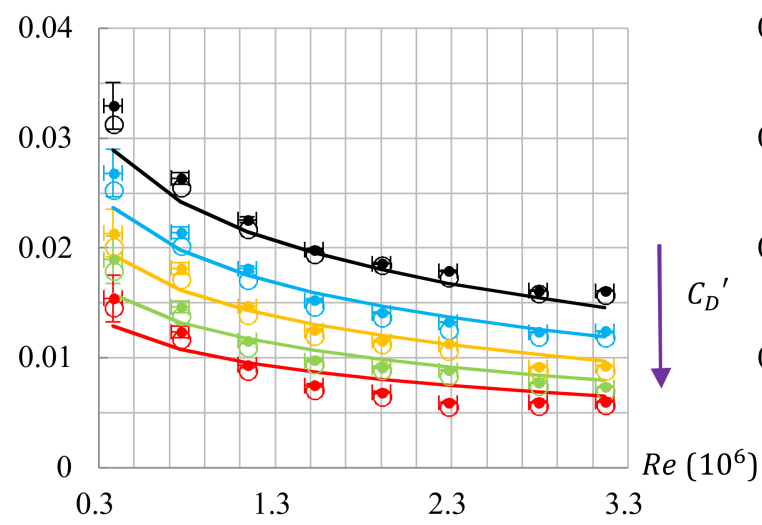

(c)

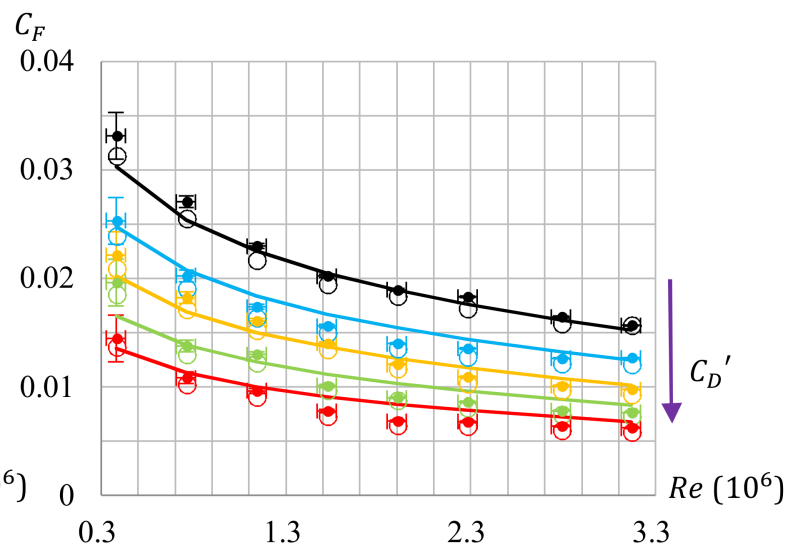

(b)

$C_{F}$

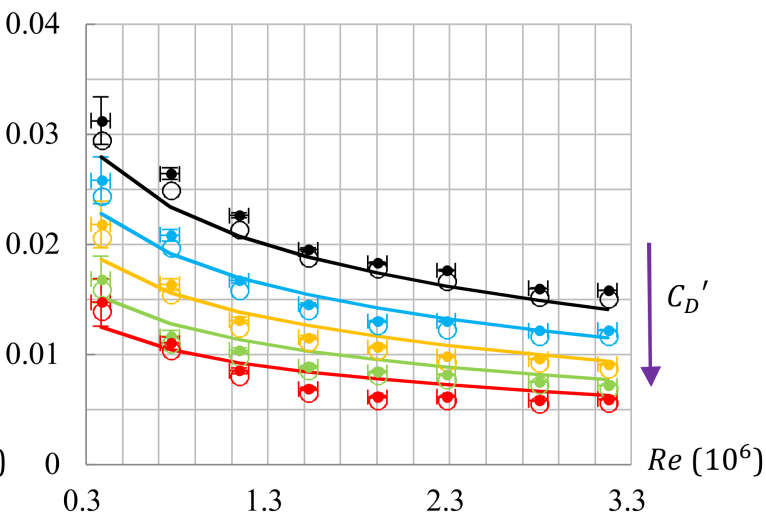

(d)

$$
\begin{aligned}
& C_{D}{ }^{\prime}=0 \quad-\operatorname{Exp} \quad \circ \quad B p \quad \longrightarrow \quad \text { Equation (12) } \quad C_{D}{ }^{\prime}=1262 \quad \bullet \quad \operatorname{Exp} \quad \circ \quad B p \quad-\quad \text { Equation (12) }
\end{aligned}
$$

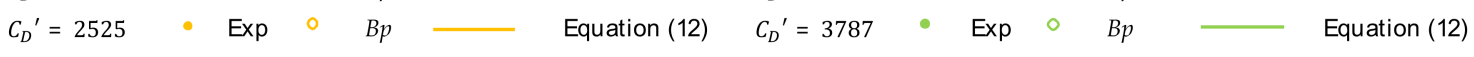

$$
\begin{aligned}
& C_{D}{ }^{\prime}=5050 \quad-\operatorname{Exp} \circ \mathrm{Bp} \longrightarrow \text { Equation (12) }
\end{aligned}
$$

Figure 12. $C_{F}(R e)$ curves: (a) $G=0.018$; (b) $G=0.036$; (c) $G=0.054$ and (d) $G=0.072$. $C_{D}{ }^{\prime}$ : through-flow coefficient; $C_{F}$ : axial thrust coefficient; $G$ : non-dimensional axial gap; $R e$ : global circumferential Reynolds number.

\subsection{D Daily and Nece Diagram}

In this study, the 2D Daily and Nece diagram is extended with centrifugal through-flow by classifying the tangential velocity profiles at $x=0.945, x=0.79$ and $x=0.57$ based on the results of numerical simulation. Currently five distinguishing lines are found, depicted in Figure 13a. Below and above the distinguishing lines are regime III (small axial gap, turbulent flow, merged boundary layers) and regime IV (large axial gap, turbulent flow, separated boundary layers), respectively. The distinguishing surface is drawn through the five distinguishing lines, shown in Figure 13b. Near the distinguishing surface, a mixing zone is found in which regime III and regime IV coexist (ignored in this study). The distinguishing surface for centripetal through-flow (Hu et al. [26]) is also plotted. 


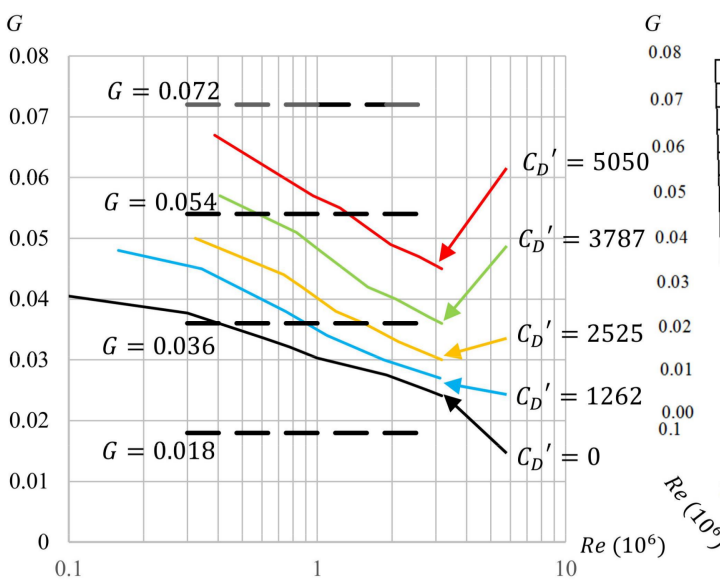

(a)

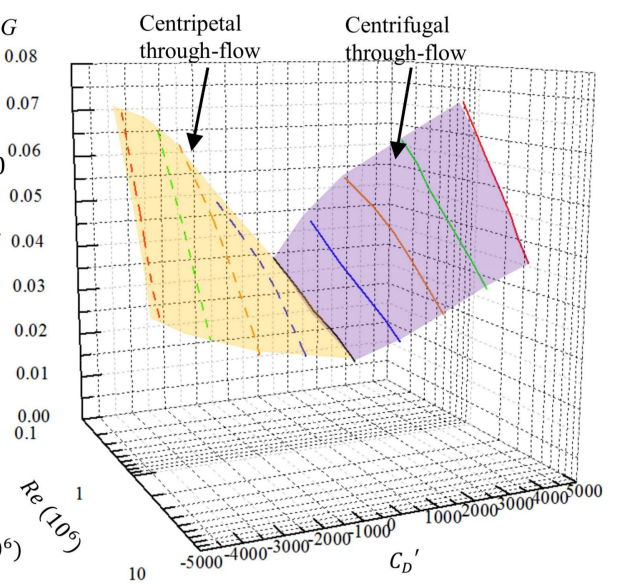

(b)

Figure 13. 3D Daily and Nece diagram: (a) Distinguishing lines and (b) Distinguishing surface. $C_{D}{ }^{\prime}$ : through-flow coefficient; G: non-dimensional axial gap; Re: global circumferential Reynolds number.

\subsection{Moment Coefficient}

According to the experimental results from Han et al. [27], the moment coefficient on the cylinder surface of the disk, noted as $C_{M c y l}$, can be estimated with Equation (13) for smooth disks.

$$
C_{M c y l}=\frac{2 \cdot\left|M_{c y l}\right|}{\rho \cdot \Omega^{2} \cdot b^{5}}=\frac{0.084 \cdot \pi \cdot t}{b \cdot\left(\lg \frac{\Omega \cdot b^{2}}{v}\right)^{1.5152}}
$$

By combining the torque measurements with the results from Daily and Nece [3] and Dorfman [28], two correlations are determined to predict the moment coefficient for a single surface of the disk, given in Equations (14) and (15). For an enclosed rotor-stator cavity, the results from Equations (14) and (15) are around 0.28 times those from the correlations by Daily and Nece [3]. Hu et al. [26] attribute the large gaps to the difference of disk surface roughness.

$$
\begin{aligned}
& \text { Regime III : } C_{M 3}=0.011 \cdot G^{-\frac{1}{6}} \cdot R e^{-\frac{1}{4}} \cdot\left[e^{\left(10^{-4} \cdot C_{D}{ }^{\prime}\right)}\right] \\
& \text { Regime IV : } C_{M 4}=0.014 \cdot G \frac{1}{10} \cdot R e^{-\frac{1}{5}} \cdot\left[e^{\left(0.6 \cdot 10^{-4} \cdot C_{D}{ }^{\prime}\right)}\right]
\end{aligned}
$$

The experimental results of $C_{M}$ are compared with those from Equations (14) and (15), depicted in Figure 14. In Figure 13a, most of the flow regimes are regime III at $G=0.018$. The experimental results of $C_{M}$ are in good accordance with those from Equation (14). When $G$ increases to 0.036 , the flow regimes shift from regime III to regime IV with the increase of $\operatorname{Re}$ for $C_{D}{ }^{\prime}=0,1262$ and 2525. The regime changes can also be found based on the experimental results of $C_{M}$. For example, at $G=0.036$ and $C_{D}{ }^{\prime}=1262$, the results of $C_{M}$ from Equation (15) rather than from Equation (14) are approaching those from torque measurements when $R e \geq 1 \times 10^{6}$. In Figure 13a, the separating point of regime III and regime IV at the same values of $G$ and $C_{D}{ }^{\prime}$ is at $R e \geq 0.75 \times 10^{6}$. The difference can be attributed to the existence of the mixing zone. For $G=0.054$ and $G=0.072$, most of the flow regimes are regime IV according to Figure 13a. The results of $C_{M}$ from Equation (15) are more coincident with those from torque measurements. The regime III may occur at small $\operatorname{Re}$ and large $C_{D}{ }^{\prime}$. The amount of $C_{M}$ increases by increasing $C_{D}{ }^{\prime}$ while it drops faster for higher Reynolds numbers $R e$ at small $G$. Compared with the cases for centripetal through-flow [26], the centrifugal through-flow will result in larger values of $C_{M}$ at the same values of $\left|C_{D}{ }^{\prime}\right|$, which is in accordance with the results of Dibelius et al. [29]. 
On the distinguishing lines (see Figure 13a), the results from Equation (14) are supposed to be equal to those from Equation (15). The results of $C_{M 3} / C_{M 4}$ at the distinguishing lines are presented in Figure 15. Most of the differences attributed to the existence of the mixing zone are very small and cover an amount lower than 5\%. According to Figure 15, Equations (14) and (15) can be used to predict the transition from regime III to regime IV with good accuracy. All the results show that the moment coefficient can be predicted with Equations (14) and (15) with the 3D diagram.

There are still some limitations of this work. All the experimental results are obtained with the smooth disk $\left(R_{z}=1 \mu \mathrm{m}\right)$. The applicability of discussed equations will become broader by introducing the impact of surface roughness of the disks in the next step. The existance of through-flow dominant region is ignored during the calculation of $C_{M}$ because it may only occur at small radius for small Reynolds numbers and large through-flow coefficient. Since the frictional torque in that region is proportional to $r_{c}{ }^{5}$ (the parameter $r_{c}$ is the radial coordinate below which the flow is dominant by through-flow), which is of small value compared with $b^{5}$, the above simplification results in small errors only. The distinguishing lines will be modified based on the measured velocity components in both tangential and radial directions in the future. The outlet geometry has a relatively large influence on the results of $K$, which deserves further investigations. The impacts of boundary conditions (at both inlet and outlet) and internal flow structures on $C_{F}$ and $C_{M}$ need to be investigated further.

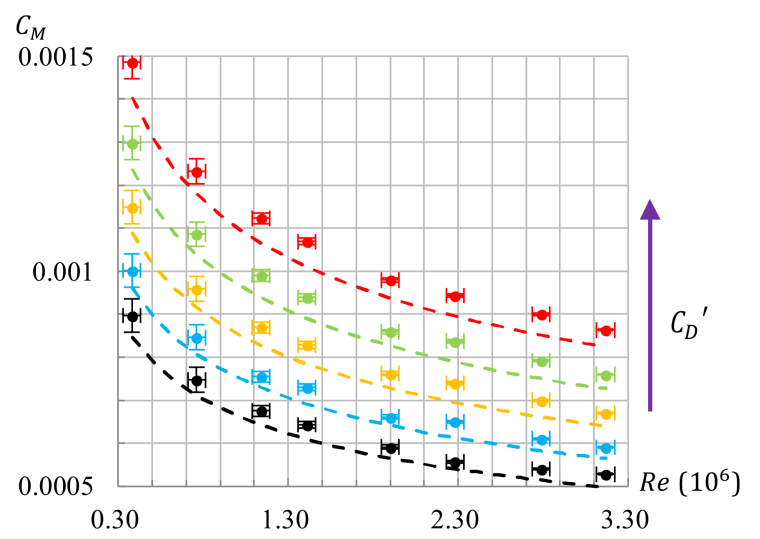

(a)

$C_{M}$

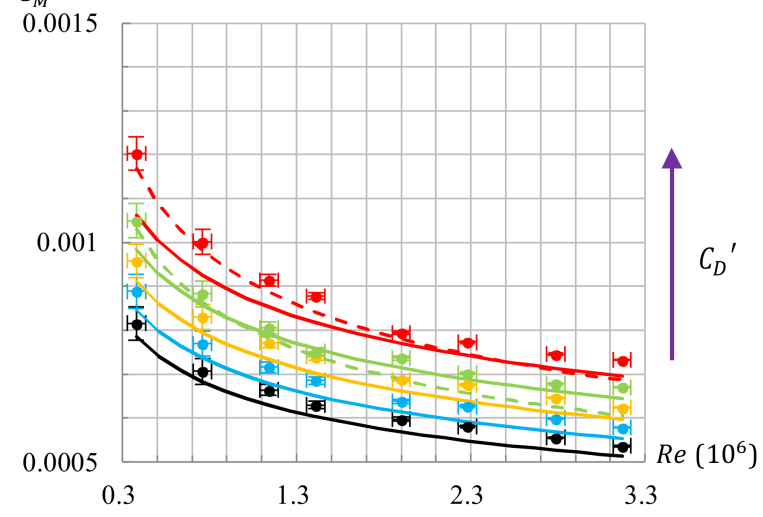

(c)

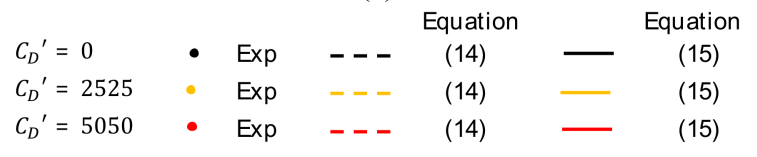

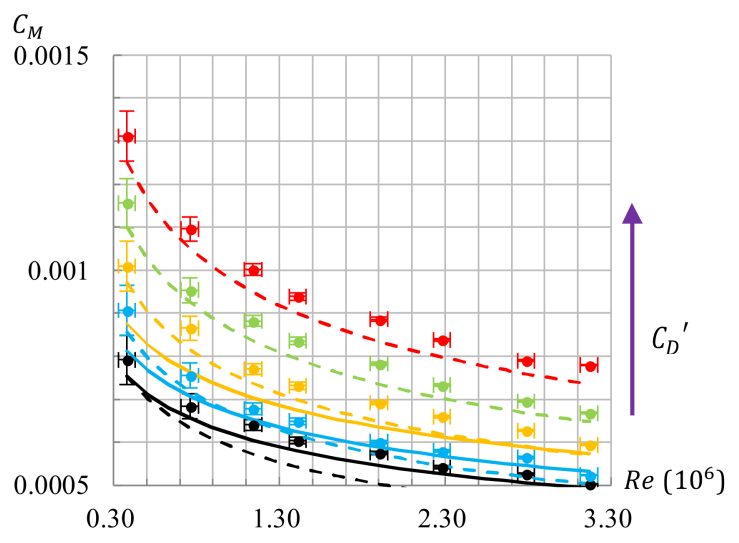

(b)

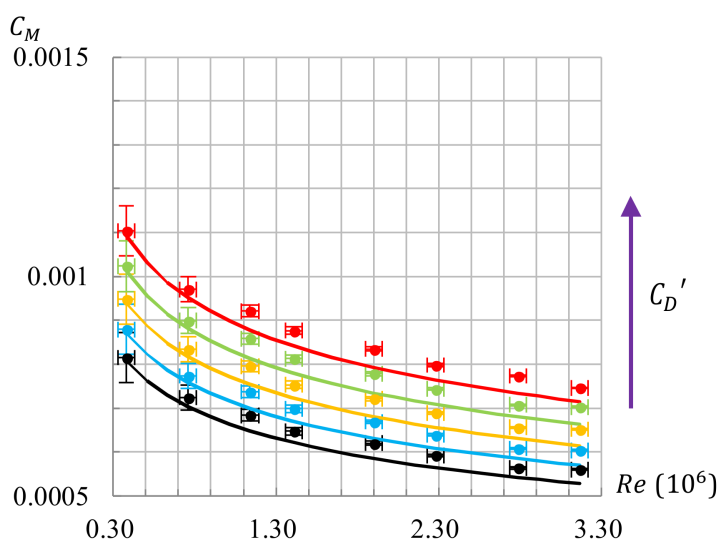

(d)

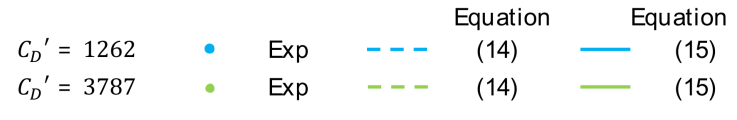

Figure 14. $C_{M}(R e)$ curves: (a) $G=0.018$; (b) $G=0.036$; (c) $G=0.054$ and (d) $G=0.072 . C_{D}{ }^{\prime}$ : through-flow coefficient; $C_{M}$ : moment coefficient; $R e$ : global circumferential Reynolds number; $G$ : non-dimensional axial gap. 


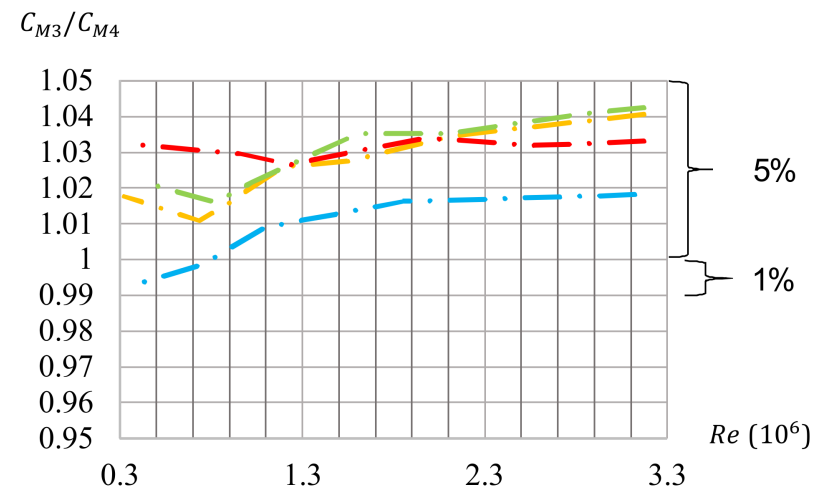

Figure 15. Results of $C_{m 3} / C_{m 4}$ at the distinguishing lines. $C_{M 3}: C_{M}$ for regime III; $C_{M 4}: C_{M}$ for regime IV. Colors: Blue: $C_{D}{ }^{\prime}=1262$, Yellow: $C_{D}{ }^{\prime}=2525$, Green: $C_{D}{ }^{\prime}=3787$; Red: $C_{D}{ }^{\prime}=5050$.

\section{Conclusions}

The influence of centrifugal through-flow on the core swirl ratio, radial pressure distribution, axial thrust and frictional torque in a rotor-stator cavity with different $G$ is strong.

Based on the pressure measurements, an empirical correlation is determined to predict the impact of $\operatorname{Re}, C_{D}{ }^{\prime}$ on $K$ when $G$ ranges from 0.018 to 0.072 .

A correlation is determined which enables to predict the influence of $G, \operatorname{Re}$ and $C_{D}{ }^{\prime}$ on the thrust coefficient $C_{F}$ for a smooth disk $\left(R_{z}=1 \mu \mathrm{m}\right)$.

By distinguishing the tangential velocity profiles for centrifugal through-flow Daily and Neceand diagram is made three-dimensional. Four distinguishing lines and the approximate distinguishing surface are presented. Two correlations are determined to predict the influence of centrifugal through-flow $C_{D}{ }^{\prime}$ on $C_{M}$ for the two zones with good accuracy for the smooth disk $\left(R_{z}=1 \mu \mathrm{m}\right)$. At the distinguishing lines, the results from the two equations are very close. The values of $C_{M}$ for centrifugal through-flow exceed those for centripetal through-flow at the same values of $\left|C_{D}{ }^{\prime}\right|$.

Using the equations for the axial thrust coefficient and the moment coefficient, the influence of the centrifugal through-flow can be better predicted with improved accuracy by designing radial pumps and turbines impellers with a smooth surface. This makes the developed correlations of significant importance for the designers.

Acknowledgments: This study is funded by CSC (China Scholarship Council) and the chair of turbomachinery at University of Duisburg-Essen.

Author Contributions: Bo Hu, Dieter Brillert, Hans Josef Dohmen and Friedrich-Karl Benra devised and designed the experiments; Hans Josef Dohmen guided the design and the construction of the test rig; Dieter Brillert was responsible for the lab safety and the risk assessment; Friedrich-Karl Benra contributed the funds for the experiments and was supervisor during the research; Bo Hu did all the measurements and wrote the paper while Friedrich-Karl Benra and Dieter Brillert reviewed the paper.

Conflicts of Interest: The authors declare no conflict of interest.

\section{Nomenclature}

\begin{tabular}{ll}
\multicolumn{2}{l}{ Latin Symbols } \\
$a$ & Hub radius \\
$B p$ & Based on the pressure calculation \\
$b$ & Outer radius of the disk \\
$C_{D}{ }^{\prime}$ & Through-flow coefficient \\
$C_{F}$ & Axial thrust coefficient \\
$C_{F f}$ & $C_{F}$ on the front surface \\
$C_{F b}$ & $C_{F}$ on the back surface \\
$C_{M}$ & Moment coefficient
\end{tabular}

$\mathrm{mm}$

$\mathrm{mm}$
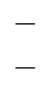
$C_{\text {Mcyl }} \quad$ Moment coefficient on the cylinder surface of the disk

$C_{M 3} \quad C_{M}$ for regime III

$C_{M 4} \quad C_{M}$ for regime IV

$C_{p} \quad$ Pressure coefficient

$C_{q r} \quad$ Local flow rate coefficient

c Constant

$\mathrm{e}_{T} \quad$ Relative error of the transducer

$e \quad$ Pressure tube number

$F_{a} \quad$ Axial thrust $\quad \mathrm{N}$

$F_{a f} \quad$ Force on the front surface of the disk $\quad \mathrm{N}$

$F_{a b} \quad$ Force on the back surface of the disk N

G Non-Dimensional axial gap -

$K \quad$ Core swirl ratio at $\zeta=0.5$

$\bar{K} \quad$ Average value of $K$ between the two adjacent pressure tubes

$M \quad$ Frictional torque Nm

$M_{c y l} \quad$ Frictional resistance on the cylinder surface of the disk $\quad \mathrm{Nm}$

$M_{r} \quad$ Measuring range $\quad \mathrm{Nm}$

$\dot{m} \quad$ Mass flow rate $\mathrm{kg} / \mathrm{s}$

$N_{D} \quad$ Uncertainty of the data acquisition system -

$N_{T} \quad$ Uncertainty of the transducer

$\Delta N \quad$ Uncertainty of the measured results

$n \quad$ Speed of rotation $\quad \mathrm{rpm}$

$p \quad$ Pressure $P a$

$p_{b} \quad$ Pressure at $r=b \quad P a$

$p^{*} \quad$ Non-dimensional pressure $\quad-$

$Q \quad$ Volumetric through-flow rate $\quad \mathrm{m}^{3} / \mathrm{s}$

Re Global circumferential Reynolds number -

$\operatorname{Re}_{\varphi} \quad$ Local circumferential Reynolds number

$r \quad$ Radial coordinate mm

$r_{c} \quad$ Radial coordinate below which the flow is dominant by through-flow $\mathrm{mm}$

$r_{e} \quad$ Radius of the shaft end $\mathrm{mm}$

$r_{\text {seal }} \quad$ Radius of the shaft seal $\mathrm{mm}$

$s \quad$ Axial gap of the front chamber $\mathrm{mm}$

$s_{b} \quad$ Axial gap of the back chamber $\mathrm{mm}$

$t \quad$ Thickness of the disk $\mathrm{mm}$

$V_{r} \quad$ Non-Dimensional radial velocity -

$V_{\varphi} \quad$ Non-Dimensional tangential velocity

$x \quad$ Non-Dimensional radial coordinate -

$z \quad$ Axial coordinate $\mathrm{mm}$

\section{Greek Symbols}

$\varepsilon \quad$ Diameter of spheres

$\mathrm{mm}$

$\zeta \quad$ Non-dimensional axial coordinate

$\mu \quad$ Dynamic viscosity of water

$v \quad$ Kinematic viscosity of water

$\rho \quad$ Density of water

$\Omega \quad$ Angular velocity of the disk

$\mathrm{N} \cdot \mathrm{s} / \mathrm{m}^{2}$

$\mathrm{m}^{2} / \mathrm{s}$

$\mathrm{kg} / \mathrm{m}^{3}$

$\mathrm{rad} / \mathrm{s}$

\section{Abbreviations}

FS Full scale

LDA Laser Doppler Anemometer

rpm Revolution per minute

SST Shear Stress Transport 


\section{References}

1. Kármán, T.V. Über laminare und turbulente Reibung. Z. Angew. Math. Mech. 1921, 1, 233-252. [CrossRef]

2. Cochran, W.G. The flow due to a rotating disk. Math. Proc. Camb. Philos. Soc. 1934, 30, 365-375. [CrossRef]

3. Daily, J.W.; Nece, R.E. Chamber dimension effects on induced flow and frictional resistance of enclosed rotating disks. J. Basic Eng. 1960, 82, 217-232. [CrossRef]

4. Daily, J.W.; Asbedian, V.V.; Ernst, W.D. Enclosed Rotating Disks with Superposed Throughflow; Report No. 64; Massachusetts Institute of Technology: Cambridge, MA, USA, 1964.

5. Bayley, F.J.; Owen, J.M. Flow between a rotating and a stationary disc. Aeronaut. Q. 1969, 20, 333-354. [CrossRef]

6. Haynes, C.M.; Owen, J.M. Heat transfer from a shrouded disk system with a radial outflow of coolant. J. Eng. Power 1975, 97, 28-35. [CrossRef]

7. Phadke, U.P.; Owen, J.M. An investigation of ingress for an air-cooled shrouded rotating disk system with radial-clearance seals. J. Eng. Power 1983, 105, 178-182. [CrossRef]

8. Launder, B.; Poncet, S.; Serre, E. Laminar, transitional, and turbulent flows in rotor-stator cavities. Annu. Rev. Fluid Mech. 2010, 42, 229-248. [CrossRef]

9. Will, B.C.; Benra, F.-K. Investigation of the Fluid Flow in a Rotor-Stator Cavity with Inward Through-Flow. In Proceedings of the ASME 2009 Fluids Engineering Division Summer Meeting, Vail, CO, USA, 2-6 August 2009.

10. Will, B.C.; Benra, F.-K.; Dohmen, H.J. Numerical and Experimental Investigation of the Flow in the Side Cavities of a Centrifugal Pump. In Proceedings of the 12th International Symposium on Transport Phenomena and Dynamics of Rotating Machinery, Honolulu, HI, USA, 17-22 February 2010.

11. Will, B.C.; Benra, F.-K.; Dohmen, H.J. Investigation of the Flow in the Side Chambers of a Centrifugal Pump with Volute Casing. In Proceedings of the 10th International Symposium on Experimental Computational Aerothermodynamics of Internal Flows, Brussels, Belgium, 4-7 July 2011.

12. Coren, D.; Childs, P.R.N.; Long, C.A. Windage sources in smooth-walled rotating disc systems. Proc. Inst. Mech. Eng. Part C 2009, 223, 873-888. [CrossRef]

13. Long, C.A.; Miles, A.L.; Coren, D.D. Windage Measurements in a Rotor Stator Cavity with Rotor Mounted Protrusions and Bolts. In Proceedings of the ASME Turbo Expo 2012, Power for Land, Sea and Air, Copenhagen, Denmark, 11-15 June 2012.

14. Barabas, B.; Clauss, S.; Schuster, S.; Benra, F.-K.; Dohmen, H.J. Experimental and Numerical Determination of Pressure and Velocity Distribution inside a Rotor-Stator Cavity at very High Circumferential Reynolds Numbers. In Proceedings of the 11th European Conference on Turbomachinery Fluid Dynamics and Thermodynamics, Madrid, Spain, 23-27 March 2015.

15. Poncet, S.; Chauve, M.-P.; Gal, P.L. Turbulent rotating disk flow with inward throughflow. J. Fluid Mech. 2005, 522, 253-262. [CrossRef]

16. Poncet, S.; Schiestel, R.; Chauve, M.-P. Centrifugal flow in a rotor-stator cavity. J. Fluid Eng. 2005, 127, 787-794. [CrossRef]

17. Poncet, S.; Chauve, M.-P.; Schiestel, R. Batchelor versus Stewartson flow structures in a rotor-stator cavity with throughflow. Phys. Fluids 2005, 17, 075110. [CrossRef]

18. Debuchy, R.; Nour, F.A.; Bois, G. On the flow behavior in rotor-stator system with superimposed flow. Int. J. Rotating Mach. 2008, 719510. [CrossRef]

19. Debuchy, R.; Rinaldi, A.; Bois, G. Contribution to an extended modeling of the core flow behaviour in a rotor-stator cavity with a superimposed radial inflow. Mech. Ind. 2015, 16, 405. [CrossRef]

20. Lock, G.D.; Yan, Y.; Newton, P.J.; Wilson, M.; Owen, J.M. Heat Transfer Measurements Using Liquid Crystal in a Pre-Swirl Rotating-Disc System. In Proceedings of the ASME Turbo Expo: Land, Sea \& Air, Atlanta, GA, USA, 16-19 June 2003.

21. Goldstein, S. On the resistance to the rotation of a disc immersed in a fluid. Math. Proc. Camb. Philos. Soc. 1935, 31, 232-241. [CrossRef]

22. Schlichting, H.; Gersten, K. Grenzschicht-Theorie; Springer: Berlin/Heidelberg, Germany, 2006.

23. Kurokawa, J.; Toyokura, T. Study on Axial Thrust of Radial Flow Turbomachinery. In Proceedings of the Second International JSME Symposium Fluid Machinery and Fluidics, Tokyo, Japan, 4-9 September 1972; pp. 31-40. 
24. Kurokawa, J.; Toyokura, T. Axial Thrust, Disc Friction Torque and Leakage Loss of Radial Flow Turbomachinery. In Proceedings of the International Conference on Pump and Turbine Design and Development, Glasgow, UK, 1-3 September 1976; Volume 1. Paper No. 5-2.

25. Kurokawa, J.; Toyokura, T. Roughness Effects on the Flow along an Enclosed Rotating Disc. Bull. JSME 1978, 21, 1725-1732. [CrossRef]

26. Hu, B.; Brillert, D.; Dohmen, H.J.; Benra, F.-K. Investigation on the flow in a rotor-stator cavity with centripetal through-flow. Int. J. Turbomach. Propuls. Power 2017, 2, 18. [CrossRef]

27. Han, H.; Gao, S.; Li, J.; Zhang, Y. Exploring fluid resistance of disk rotor based on boundary layer theory. Mech. Sci. Technol. Aerosp. Eng. 2015, 34, 1621-1625.

28. Dorfman, L.A. Resistance of a rotating rough disc. Zh. Tekh. Fiz. 1958, 28, 380-386.

29. Dibelius, G.; Radtke, F.; Ziemann, M. Experiments on friction, velocity and pressure distribution of rotating discs. In Heat and Mass Transfer in Rotating Machinery; Hemisphere Publishing Corp.: Washington, DC, USA, 1984; pp. 117-130.

(C) 2018 by the authors. Licensee MDPI, Basel, Switzerland. This article is an open access article distributed under the terms and conditions of the Creative Commons Attribution NonCommercial NoDerivatives (CC BY-NC-ND) license (https://creativecommons.org/licenses/by-nc-nd/4.0/). 